\title{
Strategies for implementing genomic selection for feed efficiency in dairy cattle breeding schemes
}

\author{
S. E. Wallén, ${ }^{* 1} M$. Lillehammer, $†$ and T. H. E. Meuwissen* \\ *Department of Animal and Aquacultural Sciences, Norwegian University of Life Sciences, PO Box 5003, 1432 Ås, Norway \\ †Nofima, PO Box 5010, 1432 Ås, Norway
}

\begin{abstract}
Alternative genomic selection and traditional BLUP breeding schemes were compared for the genetic improvement of feed efficiency in simulated Norwegian Red dairy cattle populations. The change in genetic gain over time and achievable selection accuracy were studied for milk yield and residual feed intake, as a measure of feed efficiency. When including feed efficiency in genomic BLUP schemes, it was possible to achieve high selection accuracies for genomic selection, and all genomic BLUP schemes gave better genetic gain for feed efficiency than BLUP using a pedigree relationship matrix. However, introducing a second trait in the breeding goal caused a reduction in the genetic gain for milk yield. When using contracted test herds with genotyped and feed efficiency recorded cows as a reference population, adding an additional 4,000 new heifers per year to the reference population gave accuracies that were comparable to a male reference population that used progeny testing with 250 daughters per sire. When the test herd consisted of 500 or 1,000 cows, lower genetic gain was found than using progeny test records to update the reference population. It was concluded that to improve difficult to record traits, the use of contracted test herds that had additional recording (e.g., measurements required to calculate feed efficiency) is a viable option, possibly through international collaborations.
\end{abstract}

Key words: genomic selection, feed efficiency, breeding scheme

\section{INTRODUCTION}

Improving feed efficiency is economically important because feed costs constitute the majority of the variable cost in the dairy industry. Hence, some countries have already included feed efficiency (FE) in their breeding

Received May 13, 2016.

Accepted April 18, 2017.

${ }^{1}$ Corresponding author: sini.wallen@nmbu.no goals (Pryce et al., 2014). Having access to accurate and low-cost FE measurements is difficult; hence, a lot of research efforts are devoted to this problem (de Haas et al., 2012; Veerkamp et al., 2013). The main problem in including $\mathrm{FE}$ in the breeding objective is accessing phenotypic data from a large population of daughters of progeny tested bulls. Because genomic selection can be based on fewer phenotypes than traditional selection, genomic selection would be a useful tool to improve FE, as shown by Pryce et al. (2012) and Yao et al. (2017).

Genomic selection uses dense markers covering the whole genome and addresses most of the genetic differences between the animals (Meuwissen et al., 2001). The total genetic value of selection candidates is predicted based on the estimation of SNP effects, which are estimated using reference individuals that have been genotyped and phenotyped. If the training set is large enough and relevant to the selected population, genomic selection can result in an increase in the accuracy compared with traditional selection (VanRaden et al., 2009). The number of individuals in the training set and the marker density have the greatest effect on accuracy (Hayes and Goddard, 2008; Goddard, 2009). Other factors are heritability (Daetwyler et al., 2008; Goddard, 2009), effective population size $\left(\mathrm{N}_{\mathrm{e}}\right)$, effective number of segments (Goddard, 2009), relationship between the evaluated animals and training data set (Habier et al., 2010; Wolc et al., 2011; Pszczola et al., 2012), and variance of relationships within the reference population (Habier et al., 2010). For the traits that have low heritabilities, a very large number of records will be required in the training data set to achieve high accuracies of genomic EBV in unphenotyped animals (Hayes et al., 2009). One possibility to overcome the limited size of the training set is to combine data across countries as in the global Dry Matter Initiative (de Haas et al., 2012).

In this study, stochastic simulation was used to investigate how different breeding schemes affect genetic gain without treating accuracy as a fixed value, but rather as an outcome of the simulation. By using stochastic simulation, it is also possible to study complex and 
overlapping generations and the changes in accuracy over time under different schemes (Lillehammer et al., 2011). We used residual feed intake (RFI) as a measure of FE. Residual feed intake is defined as the difference between actual and predicted feed (or energy) intake based on the requirements of the animal (Koch et al., 1963; Williams et al., 2011; Berry and Crowley, 2013). The benefits of GS are greatest when selection is for difficult to measure traits, whose recording is either too expensive or phenotypes are not easily accessible (Goddard, 2009). Both these arguments justify the use of GS for improving FE, because $\mathrm{FE}$ recording is too expensive to be carried out on large numbers of cows and the FE of milk production cannot be recorded on bulls. In this study, genomic selection strategies were developed for improving FE in Norwegian Red dairy cattle. The objectives of this research were to compare strategies for improving selection accuracy and genetic gain for FE by estimating SNP effects in experimental herds with FE recordings or in large-scale field recordings of FE. Thus, we investigate whether it is possible to use contracted test herds with additional recording for improving traits that are difficult to measure such as FE.

\section{MATERIALS AND METHODS}

Historical populations were simulated to create realistic associations between markers and genes and to create founder populations for the breeding schemes. To create these associations and a mutation-drift balance, the simulations consisted of 2,000 generations of random mating following the Fisher-Wright population model (Fisher, 1930; Wright, 1931). The founder population had an effective population size of 200 (100 males and 100 females; Hillestad et al., 2014). The simulated genome consisted of 30 pairs of chromosomes; each was $100 \mathrm{cM}$ in length. The expected number of mutations per meiosis per diploid chromosome was 2. Polymorphisms and recombinations were simulated following Sonesson and Meuwissen (2009). From the created SNP, 3,000 were randomly selected as QTL, and QTL effects were sampled from a normal distribution. Per chromosome, 500 SNP were randomly sampled to be used as genetic markers in the breeding scheme (i.e., a total of 15,000 markers).

Seven different breeding schemes were investigated: basic, milk yield and feed efficiency included in the breeding goal $(\mathbf{M Y}+\mathbf{F E})$, population wide, and 5 test herd simulations. In the basic breeding scheme, only milk yield (MY) was included in the breeding goal, whereas in $\mathrm{MY}+\mathrm{FE}$ and test herd simulations, MY and RFI as a FE trait were included in the breeding goal and they were assumed to be uncorrelated (because RFI as a measure of FE is not correlated with MY) and have equal economic weights (in all the other breeding schemes except test herd 4,000 eco25 and test herd 4,000 eco50 schemes). In the eco25 scheme, FE had $1 / 4$ of the economic weight of MY, whereas in the eco50 scheme FE had 1/2 of the economic weight of MY. In test herd simulations, FE test herds were set up (contracted), where RFI and MY were recorded. These test herds varied in total size $(500,1,000$, and 4,000) between the schemes. Basic and MY+FE schemes were investigated with both genomic selection (Meuwissen et al., 2001) and with traditional BLUP selection (ABLUP; Henderson, 1975). Test herd simulations were investigated only with genomic selection.

In the basic schemes, all cows got records only for MY at 3 yr of age (Table 1 and Figure 1). However, in the MY+FE schemes all cows had records for both MY and RFI at $3 \mathrm{yr}$ of age. In test herd schemes, the test herd females had records for both RFI and MY at $3 \mathrm{yr}$ of age, whereas other cows had records for MY only. No repeated records were assumed for any of the traits, which is conservative with respect to the amount of information that comes from recording a cow. Females were available for selection at 2, 3, 4, 5, and $6 \mathrm{yr}$ of age. All ages refer to the average generation interval that results from their mating (i.e., the actual mating occurs 9 mo earlier). Males were selected to be parents at $3 \mathrm{yr}$ of age in genomic BLUP (GBLUP) and at $6 \mathrm{yr}$ of age in ABLUP schemes. Males were progeny tested for both MY and RFI in MY+FE schemes; progeny test results were available at $6 \mathrm{yr}$ of age (Table 1 and Figure 1). However, in the basic and test herd schemes, males were progeny tested only for MY. The progeny test information was hence available when selecting sires in the ABLUP schemes, but not in the GBLUP schemes, due to the shorter generation interval. In GBLUP schemes, progeny information was used to update the reference population. One-third of the females were culled randomly every year starting when they were 3 yr old. Females in the test herds and bull calves born from elite matings were assumed genotyped in GBLUP schemes.

A base generation (generation 0) was created using the animals from the last generation of the founder population and mating them randomly. All 4,000 animals in generation 0 were assumed to be genotyped and have their own records to contribute to progeny testing in all the schemes that involved genomic selection, and those animals were used to estimate SNP effects for MY and RFI. The younger bulls were added to the simulated reference population when their daughters had records for production traits. The simulated breed- 
Table 1. Ages (yr) at which recording and selection take place ${ }^{1}$

\begin{tabular}{lccccc}
\hline Item & Age of dam & Age of sire & $\begin{array}{c}\text { Milk record } \\
\text { dam }\end{array}$ & $\begin{array}{c}\text { RFI record } \\
\text { dam }\end{array}$ & $\begin{array}{c}\text { Progeny test } \\
\text { sire }\end{array}$ \\
\hline Basic ABLUP & $2-6$ & 6 & 3 & - & $6^{2}$ \\
Basic GBLUP & $2-6$ & 3 & 3 & 3 & $6^{2}$ \\
MY+FE ABLUP & $2-6$ & 6 & 3 & 3 & $6^{3}$ \\
MY+FE GBLUP & $2-6$ & 3 & 3 & $3^{3}$ & $6^{3}$ \\
Test herd GBLUP & $2-6$ & 3 & 3 & & - \\
\hline
\end{tabular}

${ }^{1}$ Ages refer to the generation interval resulting from the mating of the parents (selected for the indicated record). $\mathrm{RFI}=$ residual feed intake; $\mathrm{ABLUP}=$ best linear unbiased prediction using pedigree relationship matrix; GBLUP = genomic best linear unbiased predictor; $\mathrm{MY}+\mathrm{FE}=$ milk yield and feed efficiency included in the breeding goal.

${ }^{2}$ Breeding goal includes only milk yield.

${ }^{3}$ Breeding goal includes both RFI and milk yield.

ing schemes closely resembled those of Lillehammer et al. (2011) where earlier progeny-tested bulls were genotyped and used to estimate SNP effects.

True breeding values (TBV) were calculated for all individuals as the sum of the QTL effects:

$$
\mathrm{TBV}_{i}=\sum_{j=1}^{\text {Number of QTL }} x_{i j 1} g_{j 1}+x_{i j 2} g_{j 2},
$$

where $x_{i j k}$ is the number of copies that individual $i$ has at the $j$ th QTL position and $k$ th QTL allele, and $g_{j k}$ is the effect of the kth QTL at the jth position, which were sampled from the normal distribution. The simulated traits, MY and RFI, were assumed to have heritabilities of 0.3 and 0.15 , respectively. Those heritabilities reflect the average heritability of milk production (Hoekstra et al., 1994; Berry et al., 2003) and the average heritability of FE traits (Berry and Crowley, 2013; Varga and Dechow, 2013).

The accuracy of the genomic breeding values was calculated, according to Sonesson and Meuwissen (2009), as the correlation between the estimated genomic breeding values and the true breeding values. Genomic breeding values were estimated by summing the marker effects:

$$
\mathrm{GEBV}_{i}=\sum_{j=1}^{n} x_{i j} a_{j}
$$

where $x_{i j}$ is the $j$ th SNP effect of individual $i, a_{j}$ is the BLUP estimate of the $j$ th SNP effect, and $n$ is the number of SNP $(15,000)$. To ensure that direct com-

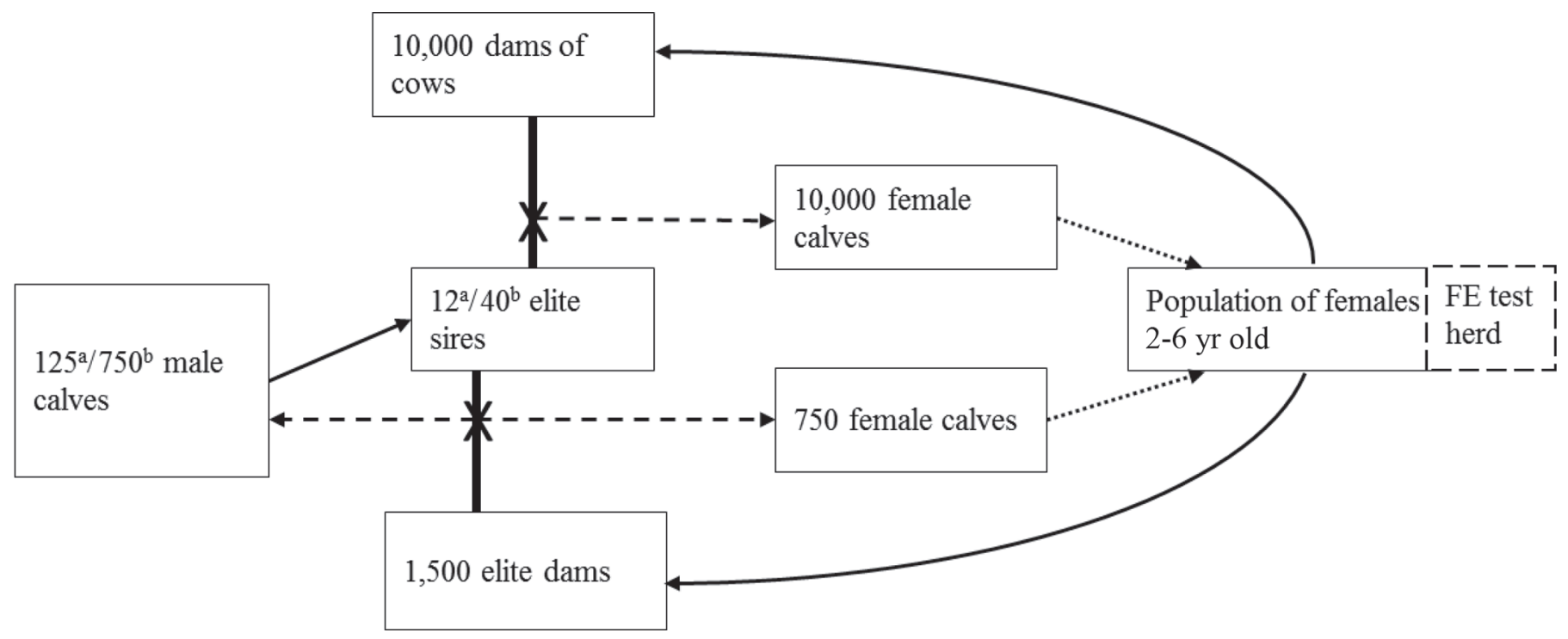

Figure 1. Overview of the breeding schemes. Solid lines with a cross represent matings, and dashed arrows represent progeny produced by the matings. Dotted arrows represent that animals move from one category to another due to aging. Solid arrows represent selection of animals. ${ }^{\text {an }}$. BLUP using pedigree relationship matrix (ABLUP) schemes, 125 male calves were progeny tested and 12 elite sires were selected. ${ }^{b}$ In genomic BLUP schemes, 750 male calves were progeny tested and 40 elite sires were selected. $\mathrm{FE}=$ feed efficiency. 
parison between traditional and genomic EBV was possible, all EBV were scaled so that $b=1$, where $b=\operatorname{Cov}\left[\mathrm{TBV}_{i} ;(\mathrm{G}) \mathrm{EBV}_{i}\right] / \operatorname{Var}\left[(\mathrm{G}) \mathrm{EBV}_{i}\right]$. This is important for the selection of females, which is across genomic EBV and traditional EBV for some of the schemes.

Phenotypes were simulated by adding a normally distributed random error term to the true breeding value:

$$
P_{i}=\mathrm{TBV}_{i}+\varepsilon_{i}
$$

where $\varepsilon_{i}$ is an error term for animal $i$, which was normally distributed $\left(0, \sigma_{e}^{2}\right)$. To express the results in genetic standard deviations and create phenotypic records with the desired heritability, the genetic variance $\left(\sigma_{g}^{2}\right)$ was scaled to 1 for both of the traits and the residual variance $\left(\sigma_{e}^{2}\right)$ was adjusted following Sonesson and Meuwissen (2009).

The value of 1 genetic standard deviation of $\mathrm{MY}$ was arbitrarily set to 100 monetary units. When the economic value of RFI equaled that of MY, a genetic standard deviation of RFI also represented 100 monetary units. In schemes with reduced economic values for RFI, eco50 and eco25 schemes, 1 genetic standard deviation of RFI represented 50 and 25 monetary units, respectively.

The BLUP method (Meuwissen et al., 2001) was used for the estimation of marker effects. The statistical model used to estimate individual marker effects was

$$
y_{i}=\mu+\sum_{j=1}^{n} X_{i j} a_{j}+e_{i}
$$

where $y_{i}$ is the record of individual $i ; \mu$ is the overall mean; $X_{i j}$ is the marker genotype; $a_{j}$ is the random effect of the $j$ th marker, with variance equal to the total genetic variance divided by the number of markers; and $e_{i}$ is a random residual.

Simulated population sizes were smaller than those of the real Norwegian Red dairy cattle population to make stochastic simulation computationally possible. Population sizes were rescaled as described by Lillehammer et al. (2011), so that selection steps for conformation traits of bulls and bull dams were not considered in the simulation and selection intensities for the included traits were maintained at realistic levels when population size was reduced. The ABLUP schemes were designed to mimic the breeding structure of Norwegian Red before implementation of genomic selection, whereas the GBLUP schemes mimic the cur- rent breeding structure of Norwegian Red after genomic selection was implemented (Figure 1; Lillehammer et al., 2011).

For each scheme, 50 replicates were run and simulations were performed over a 20-yr period. Genetic gain and selection accuracy for males and females were reported as an average over yr 10 to 20 of the simulations. In all the schemes, total genetic gain was calculated by summing up the genetic gain (in monetary units) for MY and RFI. When results of the simulation study are reported, omitting the first years of the simulation avoids the problem of the non-steady-state population structure at the start of the scheme, where all animals are of the same age and all base generation animals are assumed to be genotyped and progeny tested, which affects early simulation results.

\section{RESULTS}

Figure 2 shows the total genetic gain (in monetary units) for MY and RFI when RFI was included in the breeding scheme. The highest total genetic gain was found when using the MY+FE GBLUP scheme, where bulls were progeny tested for both traits. The ABLUP schemes gave lower total genetic gain when compared with a similar GBLUP scheme. Increasing the number of cows in the test herds caused an increase in genetic gain. Genotyping 500 or 1,000 cows in test herds resulted lower genetic gain than using progeny test records to update the reference population. However, a test herd size of 4,000 cows gave slightly lower genetic gain than the MY+FE GBLUP scheme. Using smaller economic values for FE in test herd 4,000 GBLUP eco schemes decreased the total genetic gain.

As expected, the basic scheme gave the highest genetic gain for MY of the ABLUP schemes (Table 2 and Figure 3), and GBLUP schemes gave higher genetic gain for MY than similar ABLUP schemes. As expected, introducing a second trait in the breeding goal reduced genetic gain for MY. This is due to the fact that if selection pressure is devoted to more traits, the progress for each of the original traits decreases.

The highest genetic gain for RFI was reached using the MY+FE GBLUP scheme (Table 2 and Figure 4), where all cows had RFI records. Obtaining RFI records from test herds of limited size gave less gain for RFI, but increasing the number of genotyped cows in the test herd schemes increased the genetic gain for RFI. At a test herd size of 4,000 genotyped cows, the genetic gain for RFI was very similar to obtaining records from all cows in the population. As expected, test herd 4,000 GBLUP eco schemes gave lower genetic gain for RFI 


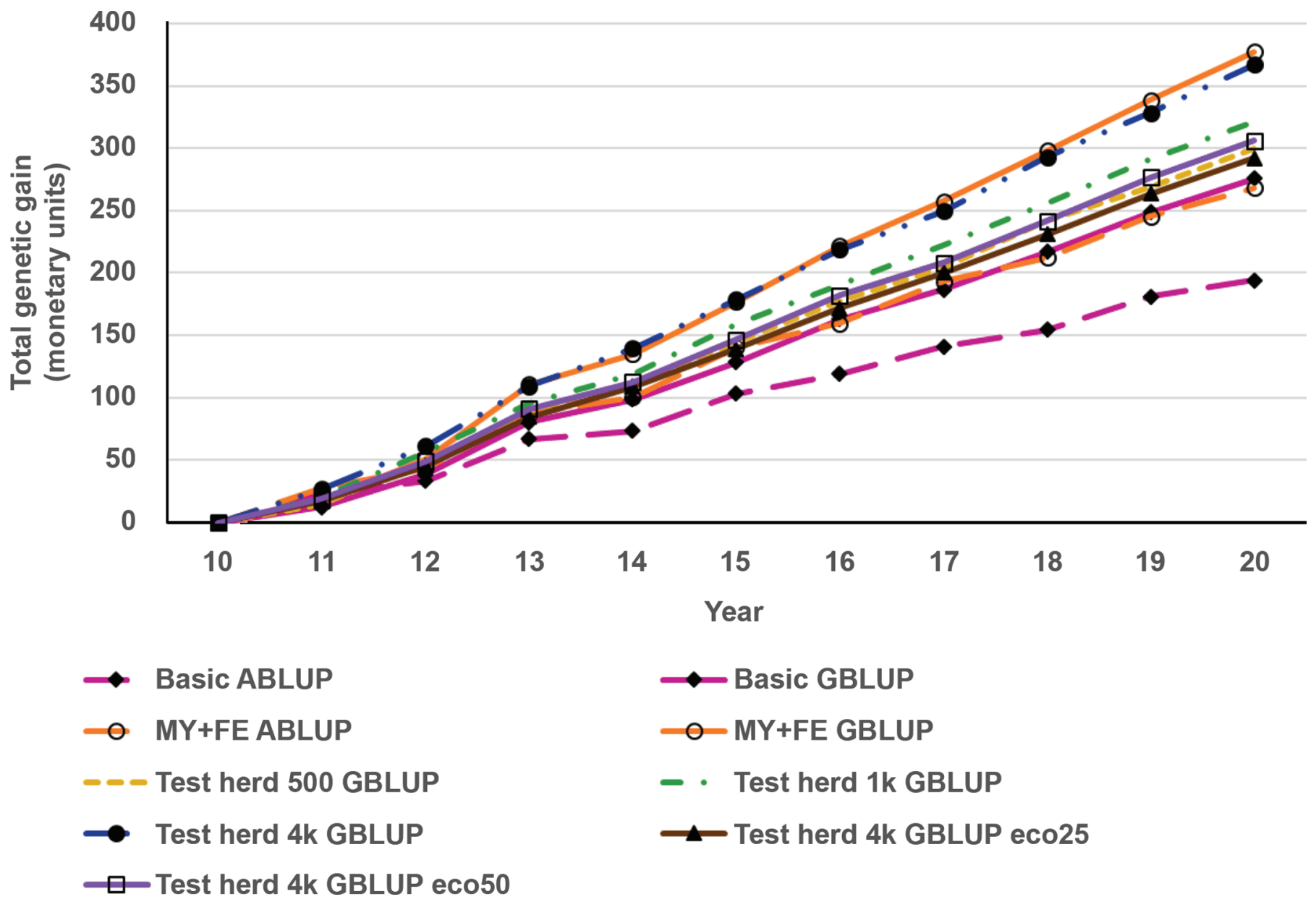

Figure 2. Total genetic gain measured as monetary units relative to yr 10 onward. In the basic schemes, genetic gain is only for milk yield, whereas in the schemes with milk yield and feed efficiency included in the breeding goal (MY+FE) and test herd schemes, genetic gain is the total genetic gain when summing up the genetic gain for both residual feed intake (RFI) and milk yield. In eco25 and eco50 schemes, 1 genetic $\mathrm{SD}$ of RFI represented 25 and 50 monetary units, respectively. ABLUP $=$ BLUP using pedigree relationship matrix; GBLUP $=$ genomic BLUP. Color version available online.

Table 2. Average genetic gain $(\Delta \mathrm{G})$ as monetary units with $\mathrm{SE}$ when $\mathrm{h}^{2}=0.3$ for milk yield and $\mathrm{h}^{2}=0.15$ for residual feed intake ${ }^{1}$

\begin{tabular}{|c|c|c|c|c|}
\hline \multirow[b]{2}{*}{ Breeding scheme $^{2}$} & \multicolumn{2}{|c|}{ Milk yield } & \multicolumn{2}{|c|}{ Residual feed intake } \\
\hline & $\begin{array}{l}\text { ABLUP, } \\
\qquad \mathrm{G}\end{array}$ & $\begin{array}{l}\text { GBLUP } \\
\quad \Delta \mathrm{G}\end{array}$ & $\begin{array}{l}\text { ABLUP, } \\
\Delta \mathrm{G}\end{array}$ & $\begin{array}{c}\text { GBLUP, } \\
\Delta \mathrm{G}\end{array}$ \\
\hline Basic & $19.64(0.2)$ & $28.52(0.2)$ & $-^{3}$ & ${ }^{3}$ \\
\hline $\mathrm{MY}+\mathrm{FE}$ & $14.76(0.2)$ & $21.74(0.3)$ & $12.45(0.2)$ & $17.28(0.3)$ \\
\hline Test herd 500 & - & $18.37(0.3)$ & - & $12.49(0.2)$ \\
\hline Test herd 1,000 & - & $18.99(0.3)$ & - & $13.88(0.3)$ \\
\hline Test herd 4,000 & - & $20.06(0.3)$ & - & $17.18(0.2)$ \\
\hline Test herd 4,000 eco25 & - & $28.74(0.06)$ & - & $1.23(0.06)$ \\
\hline Test herd 4,000 eco50 & - & $26.08(0.15)$ & - & $5.21(0.13)$ \\
\hline
\end{tabular}

${ }^{1}$ Average of genetic gain measured as genetic SD of yr 10 to 20 . The value of 1 genetic SD of milk yield was arbitrarily set to 100 monetary units. In eco25 and eco50 schemes, 1 genetic SD of residual feed intake represented 25 and 50 monetary units, respectively.

${ }^{2}$ Milk yield and feed efficiency included in the breeding goal (MY+FE) and test herd schemes include both milk yield and residual feed intake in the breeding goal; the basic scheme includes only milk yield.

${ }^{3}$ Residual feed intake is not included in the basic scheme. 


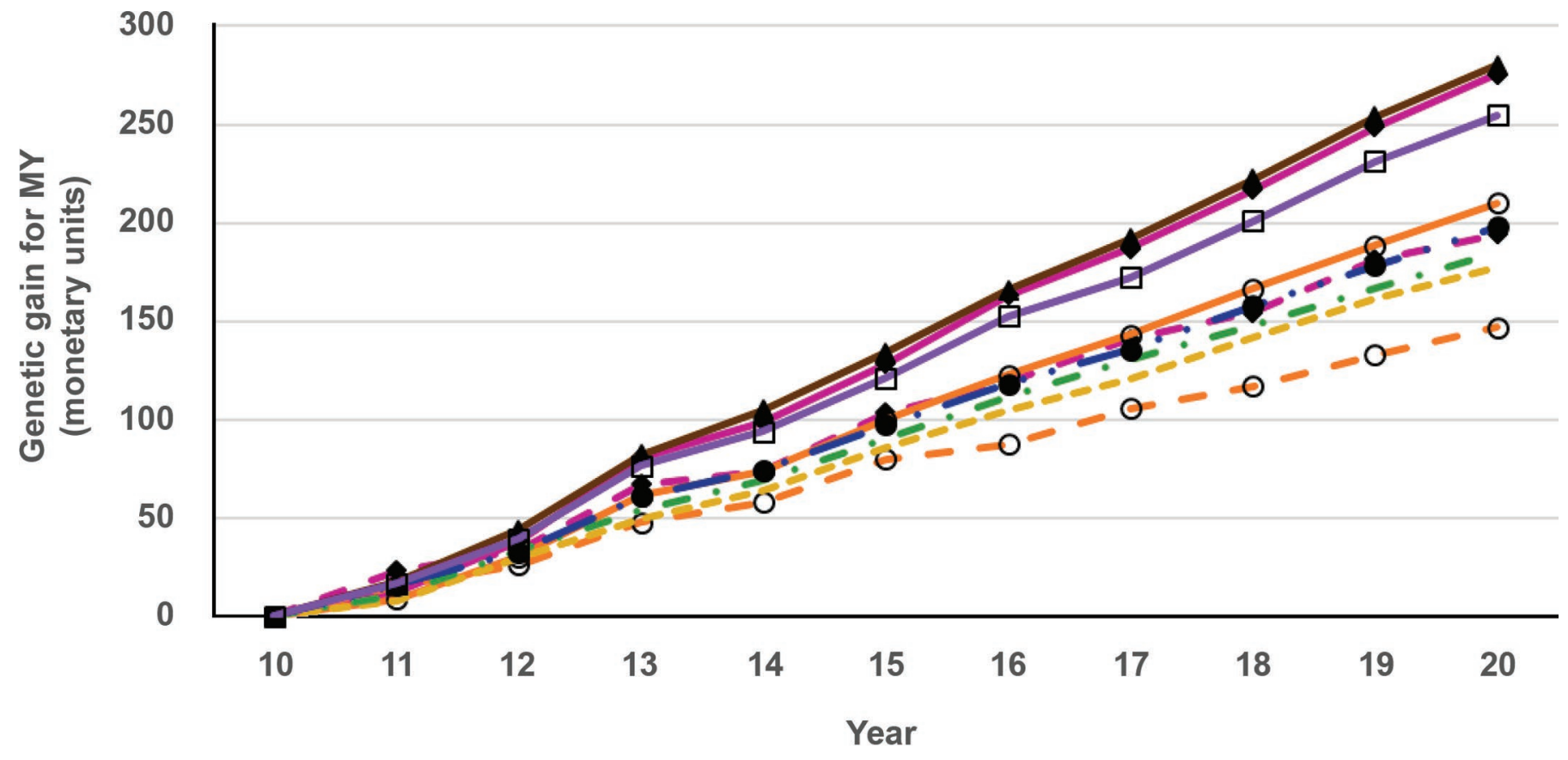

\section{- Basic ABLUP \\ $-\mathrm{O}-\mathrm{MY}+\mathrm{FE}$ ABLUP \\ - - Test herd 500 GBLUP \\ $\rightarrow \quad$ Test herd 4k GBLUP \\ —- Test herd 4k GBLUP eco50}

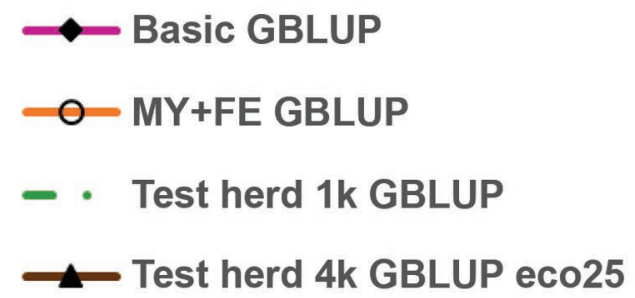

Figure 3. Genetic gain for milk yield (MY) measured as monetary units relative to yr 10 onward. In the basic schemes, genetic gain is only for MY, whereas in the schemes with MY and feed efficiency included in the breeding goal (MY+FE) and test herd schemes, genetic gain is the total genetic gain when summing up the genetic gain for both residual feed intake (RFI) and MY. In eco25 and eco50 schemes, 1 genetic SD of RFI represented 25 and 50 monetary units, respectively. ABLUP = BLUP using pedigree relationship matrix; GBLUP = genomic BLUP. Color version available online.

than other GBLUP schemes where RFI was included, which is due to the smaller economic value for RFI in eco schemes.

Selection accuracies for males ranged from 0.65 to 0.79 in GBLUP schemes and 0.94 to 0.96 in ABLUP schemes (Figure 5 and Table 3 ). Using lower economic values for RFI in the test herd, 4,000 GBLUP eco schemes slightly increased the selection accuracy for males (Figure 5). However, the selection accuracy for females was approximately 0.6 in all the other schemes except the test herd schemes (Table 3 ). The test herd scenarios caused a decrease in the selection accuracy for females because only a fraction of the females obtained RFI records. However, increasing the test herd size resulted in an increase in the female selection accuracy. The highest selection accuracy for females was reached using basic schemes, where the breeding goal included only MY.

\section{DISCUSSION}

This study compared different designs of implementing genomic selection and traditional BLUP selection for the genetic improvement of FE, and investigated how genetic gain accumulates over time and the selection accuracies that are achievable through the addition of genotyped females in the reference population. We used RFI as a FE trait because it is by definition the component of feed intake that is uncorrelated with MY. Practical breeding schemes may select directly for MY and against feed intake, but also here only the component that is uncorrelated with MY will be 


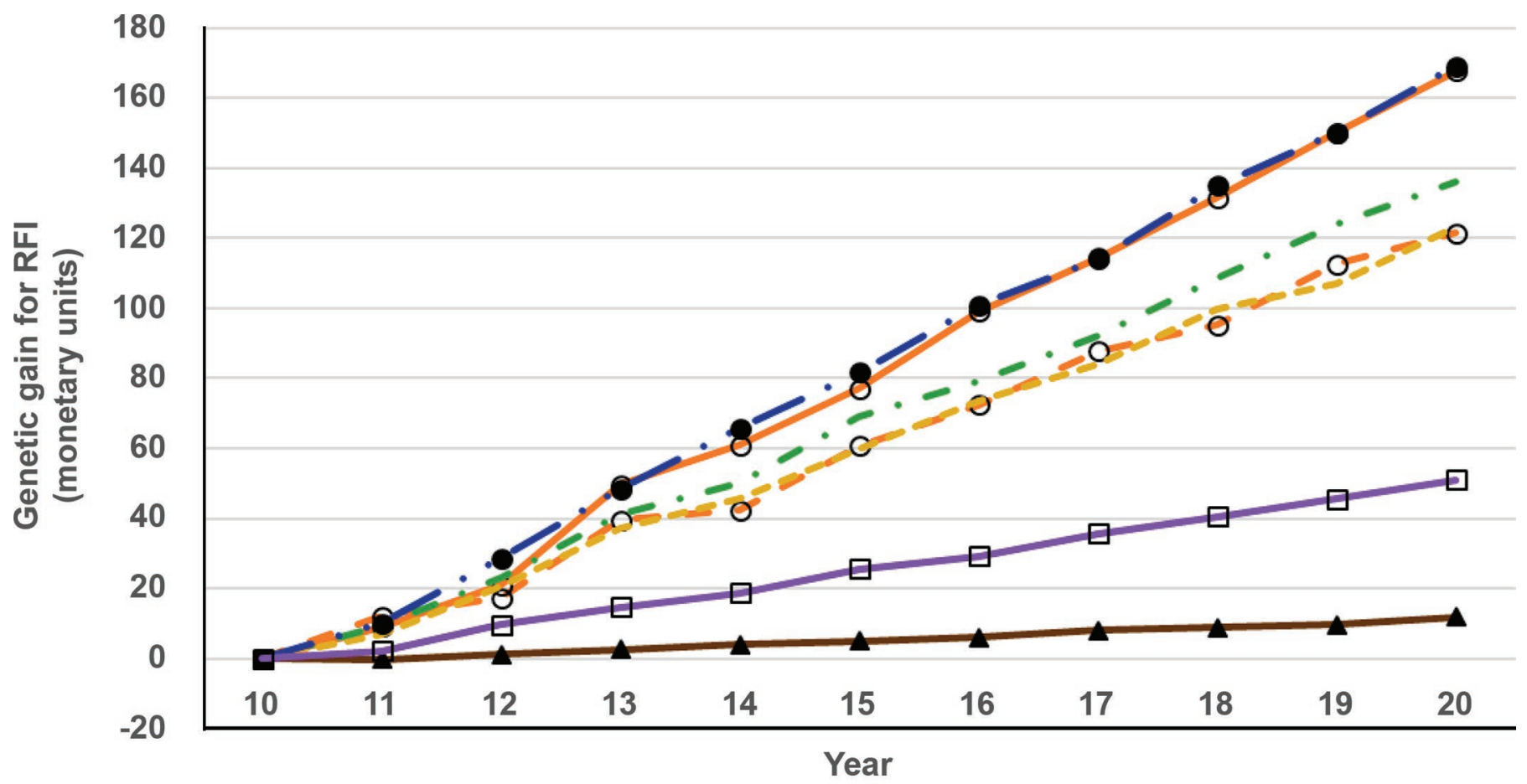

$-\mathrm{O}-\mathrm{MY}+\mathrm{FE}$ ABLUP

--- Test herd 500 GBLUP

$\rightarrow$ Test herd 4k GBLUP

$\square$ Test herd 4k GBLUP eco50
- MY+FE GBLUP

- Test herd 1k GBLUP

— Test herd 4k GBLUP eco25

Figure 4. Genetic gain for residual feed intake (RFI) measured as monetary units relative to yr 10 onward. In the schemes with milk yield and feed efficiency included in the breeding goal (MY+FE) and test herd schemes, genetic gain is the total genetic gain when summing up the genetic gain for both RFI and milk yield. In eco25 and eco50 schemes, 1 genetic SD of RFI represented 25 and 50 monetary units, respectively. $\mathrm{ABLUP}=\mathrm{BLUP}$ using pedigree relationship matrix; GBLUP = genomic BLUP. Color version available online.

reduced, whereas the component of feed intake that is associated with MY will increase together with the general increase in MY.

Table 3 showed that it is possible to achieve high selection accuracies for males when including $\mathrm{FE}$ in GBLUP schemes. This can be done either by obtaining phenotypes from all cows in the population and hence get progeny information for genotyped bulls that can be used to update a reference population, or by updating the reference population through genotyping of cows with records. The latter will be preferable if genotyping is cheap compared with phenotyping. These results are in line with Chesnais et al. (2016) who found, in real data, that the accuracies of genomic selection for $\mathrm{FE}$ varied between 0.45 and 0.58 . When using genomic selection to improve low heritability traits, the number of records in the reference population has to be suffi- ciently large to achieve high selection accuracies (Hayes et al., 2009). Our study showed that 4,000 cows had to be phenotyped and genotyped every year to achieve a similar selection accuracy of genomic selection as if all cows were phenotyped, but when only bulls are genotyped.

Females were always selected on ABLUP, except in test herd schemes, where the genotyped test-herd females obtained genomic breeding values. The female selection accuracy were first of all affected by whether the females had records for the trait under selection or not, giving higher female selection accuracy for schemes where phenotypes for all traits under selection were available for the entire cow population (Table 3). When test herds were used, the females belonging to these herds will have more accurate breeding values than the cows outside the test herds, due to their phe- 
Table 3. Average selection accuracy of yr 10 to 20 for males (M) and females (F) in the total breeding goal with SE when $\mathrm{h}^{2}=0.3$ for milk yield and $\mathrm{h}^{2}=0.15$ for residual feed intake (RFI)

\begin{tabular}{lcccc}
\hline Breeding scheme $^{1}$ & $\begin{array}{c}\text { ABLUP, } \\
\text { Accuracy M }\end{array}$ & $\begin{array}{c}\text { GBLUP, } \\
\text { Accuracy M }\end{array}$ & $\begin{array}{c}\text { ABLUP, } \\
\text { Accuracy F }\end{array}$ & $\begin{array}{c}\text { GBLUP, } \\
\text { Accuracy F }\end{array}$ \\
\hline Basic & $0.96(0.0005)$ & $0.75(0.002)$ & $0.61(0.002)$ & $0.62(0.001)$ \\
MY+FE & $0.94(0.0007)$ & $0.72(0.002)$ & $0.58(0.001)$ & $0.59(0.001)$ \\
Test herd 500 & - & $0.65(0.002)$ & - & $0.21(0.003)$ \\
Test herd 1,000 & - & $0.67(0.002)$ & - & $0.24(0.003)$ \\
Test herd 4,000 & - & $0.75(0.001)$ & - & $0.42(0.003)$ \\
Test herd 4,000 eco25 & - & $0.79(0.001)$ & - & $0.60(0.002)$ \\
Test herd 4,000 eco50 & - & $0.78(0.002)$ & - & $0.53(0.004)$ \\
\hline
\end{tabular}

${ }^{1}$ Milk yield and feed efficiency included in the breeding goal (MY+FE) and test herd schemes include both milk yield and residual feed intake; the basic scheme includes only milk yield. In eco25 and eco50 schemes, 1 genetic SD of RFI represented 25 and 50 monetary units, respectively.

notypes and genotypes. The female selection accuracy will hence depend on the fraction of the cows that are included in the test herds.

Genetic gain will depend on both male and female selection accuracy, although the male selection accuracy has the greatest effect because of the higher intensity of selection. Genetic gain was therefore similar in test herd 4,000 GBLUP as in MY+FE GBLUP, reflecting the similar accuracy of the genomic breeding values in the 2 schemes. The small advantage of MY+FE GBLUP,

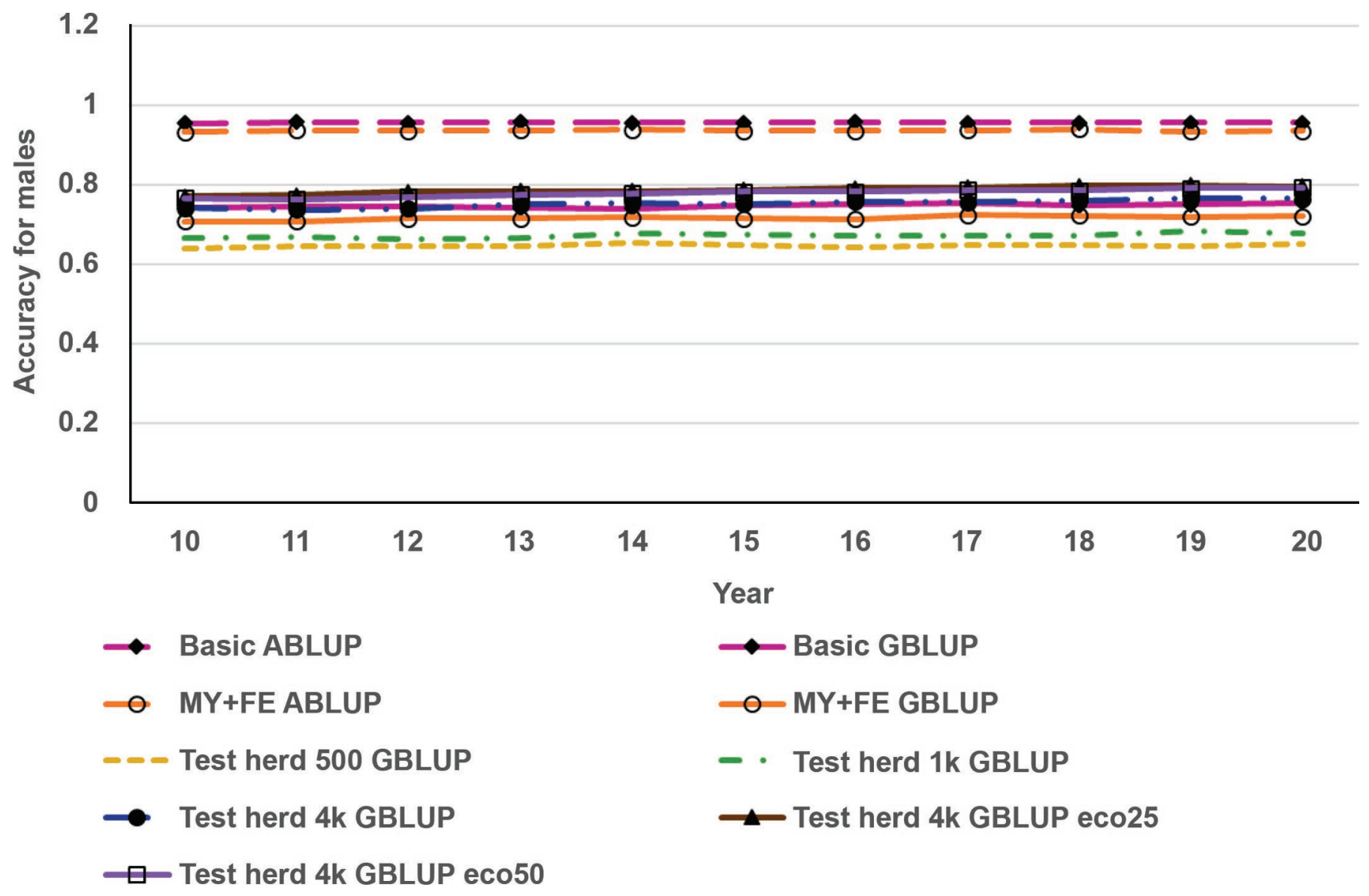

Figure 5. Selection accuracy for males relative to yr 10 onward. In the basic schemes, genetic gain is only for milk yield, whereas in the schemes with milk yield and feed efficiency included in the breeding goal (MY+FE) and test herd schemes, genetic gain is the total genetic gain when summing up the genetic gain for both residual feed intake (RFI) and milk yield. In eco25 and eco50 schemes, 1 genetic SD of RFI represented 25 and 50 monetary units, respectively. ABLUP = BLUP using pedigree relationship matrix; GBLUP = genomic BLUP. Color version available online. 
compared with test herd 4,000 GBLUP, may increase if a more intense selection of females is used. However, if selection of females were also based on genomic selection, this difference could disappear, as the fraction of the female population with RFI phenotypes becomes less important. The general level of the genetic gains agrees with those found by Lillehammer et al. (2011).

We also investigated how the reduced economic values for RFI affects the genetic gain and the accuracy of selection by comparing the test herd 4,000 GBLUP at a half and a quarter of its original economic value of RFI. As expected, test herd 4,000 GBLUP eco schemes gave higher genetic gain for MY and lower genetic gain for RFI compared with other schemes. Lower economic values for RFI increased the selection accuracy of males and especially females because many more phenotypes were available for MY than for RFI in the test herd scheme. Total genetic gain was reduced for the schemes with lower economic values for RFI. To build up test herds to facilitate genomic selection for traits with low economic value might hence not be economically defendable, as the expected gain is sensitive to the weight put on these traits.

In these simulations, we assumed a large reference population at the start of the breeding scheme, which might be optimistic. However, Figure 5 shows that genomic selection accuracies during yr 10 to 20 remain stable, implying that the gain of accuracy due to the genotyping of new relevant reference animals is compensated for old reference animals becoming less relevant (i.e., the start reference population is becoming less and less relevant during yr 10 to 20). The results of Table 3 and Figure 5 show that if progeny testing for $\mathrm{FE}$ is not feasible, genotyping females in test herds that enter a reference population may compensate for the lack of progeny testing. However, this requires the genotyping and phenotyping of 4,000 test females annually because smaller test herd sizes resulted in markedly reduced genetic gains. Obtaining large amounts of animals with multiple recordings is possible using for example collaboration between countries (de Haas et al., 2012; Veerkamp et al., 2013) or milk mid-infrared predicted FE records. McParland et al. (2014) showed that mid-infrared spectrometry of milk could be used to predict RFI as a measure of FE in lactating dairy cows. Because individual animal milk samples are routinely taken as part of the dairy herd management, using these samples to also predict feed intake and efficiency would be cost effective and a relatively undemanding approach to obtain large numbers of FE phenotypes.

In this study, we used RFI as a measure of FE. However, earlier studies showed that weak unfavorable genetic correlations exist between RFI and fertility (Vallimont et al., 2013). This is probably due to the mathematical similarity in the calculations of RFI and energy balance and a failure to account correctly for body tissue mobilization, which might lead to selection for a trait that is similar to selecting for a negative energy balance (Pryce et al., 2014). Therefore, genetic correlations with other traits (especially fertility traits) must be accounted for when including RFI into the breeding scheme (Pryce et al., 2014). That is, a multitrait selection index where genetic correlations with other traits are properly accounted for is required if RFI is to be included in the selection objective.

Feed efficiency is a trait that is difficult to measure and as such is difficult to include in the routine progeny test evaluations. Our results show that for these kind of traits, the use of rather large contracted test herds with additional recording is a viable option. This strategy would give close to similar accuracy of genomic selection as recording this trait in the whole female population. This implies that the male selection, which is the most intense selection, would be as effective with contracted test herds of genotyped females as when a routine progeny test would be performed for this trait, as long as a sufficient number of cows $(4,000)$ is included in the test herds.

\section{ACKNOWLEDGMENTS}

The helpful comments of 3 anonymous reviewers are gratefully acknowledged. This project was funded by the Norwegian Research Council (project no. 225233/ E40), the breeding and AI organization GENO ( $\AA$ s, Norway), and the Norwegian dairy foods company TINE (Oslo, Norway). Computations were performed at the Abel cluster at the University of Oslo with support from the NOTUR (Oslo, Norway) project (project no. nn4676k).

\section{REFERENCES}

Berry, D. P., F. Buckley, P. Dillon, R. D. Evans, M. Rath, and R. F. Veerkamp. 2003. Genetic relationships among body condition score, body weight, milk yield, and fertility in dairy cows. J. Dairy Sci. 86:2193-2204.

Berry, D. P., and J. J. Crowley. 2013. CELL BIOLOGY SYMPOSIUM: Genetics of feed efficiency in dairy and beef cattle. J. Anim. Sci. 91:1594-1613.

Chesnais, J. P., T. A. Cooper, G. R. Wiggans, M. Sargolzaei, J. E. Pryce, and F. Miglior. 2016. Using genomics to enhance selection of novel traits in North American dairy cattle. J. Dairy Sci. 99:2413-2427.

Daetwyler, H. D., B. Villanueva, and J. A. Woolliams. 2008. Accuracy of predicting the genetic risk of disease using a genome-wide approach. PLoS One 3:e3395.

de Haas, Y., M. P. L. Calus, R. F. Veerkamp, E. Wall, M. P. Coffey, H. D. Daetwyler, B. J. Hayes, and J. E. Pryce. 2012. Improved accuracy of genomic prediction for dry matter intake of dairy cattle from combined European and Australian data sets. J. Dairy Sci. 95:6103-6112. 
Fisher, R. A. 1930. The Genetical Theory of Natural Selection. Clarendon Press, Oxford, UK.

Goddard, M. E. 2009. Genomic selection: Prediction of accuracy and maximization of long-term response. Genetica 136:245-257.

Habier, D., J. Tetens, F.-R. Seefried, P. Lichtner, and G. Thaller 2010. The impact of genetic relationship information on genomic breeding values in German Holstein cattle. Genet. Sel. Evol. 42:5.

Hayes, B. J., P. J. Bowman, A. J. Chamberlain, and M. E. Goddard. 2009. Invited review: Genomic selection in dairy cattle: Progress and challenges. J. Dairy Sci. 92:433-443.

Hayes, B. J., and M. E. Goddard. 2008. Technical note: Prediction of breeding values using marker-derived relationship matrices. J. Anim. Sci. 86:2089-2092.

Henderson, C. R. 1975. Best linear unbiased estimation and prediction under a selection model. Biometrics 31:423-447.

Hillestad, B., J. A. Woolliams, T. Meuwissen, D. I. Våge, and G. Klemetsdal. 2014. Estimating rate of inbreeding and effective population size using genomic data in Norwegian Red cattle. Poster 470 in Proceedings of the 10th World Congress of Genetics Applied to Livestock Production. Accessed Sep. 20, 2016. https:// asas.org/wcgalp-proceedings/genetic-improvement-programsselection-using-molecular-information-(posters)

Hoekstra, J., A. W. van der Lugt, J. H. J. van der Werf, and W. Ouweltjes. 1994. Genetic and phenotypic parameters for milk production and fertility traits in upgraded dairy cattle. Livest. Prod. Sci. 40:225-232.

Koch, R. M., L. A. Swiger, D. Chambers, and K. E. Gregory. 1963. Efficiency of feed use in beef cattle. J. Anim. Sci. 33:486-494.

Lillehammer, M., T. H. E. Meuwissen, and A. K. Sonesson. 2011. A comparison of dairy cattle breeding designs that use genomic selection. J. Dairy Sci. 94:493-500.

McParland, S., E. Lewis, E. Kennedy, S. G. Moore, B. McCarthy, M. O'Donovan, S. T. Butler, J. E. Pryce, and D. P. Berry. 2014 Mid-infrared spectrometry of milk as a predictor of energy intake and efficiency in lactating dairy cows. J. Dairy Sci. 97:5863-5871.

Meuwissen, T. H. E., B. J. Hayes, and M. E. Goddard. 2001. Prediction of total genetic value using genome-wide dense marker maps. Genetics 157:1819-1829.

Pryce, J. E., J. Arias, P. J. Bowman, S. R. Davis, K. A. Macdonald, G. C. Waghorn, W. J. Wales, Y. J. Williams, R. J. Spelman, and B. J. Hayes. 2012. Accuracy of genomic predictions of residual feed intake and 250-day body weight in growing heifers using 625,000 single nucleotide polymorphism markers. J. Dairy Sci. 95:2108-2119.

Pryce, J. E., W. J. Wales, Y. de Haas, R. F. Veerkamp, and B. J. Hayes. 2014. Genomic selection for feed efficiency in dairy cattle. Animal 8:1-10.
Pszczola, M., T. Strabel, J. A. M. van Arendonk, and M. P. L. Calus. 2012. The impact of genotyping different groups of animals on accuracy when moving from traditional to genomic selection. J. Dairy Sci. 95:5412-5421.

Sonesson, A. K., and T. H. E. Meuwissen. 2009. Testing strategies for genomic selection in aquaculture breeding programs. Genet. Sel. Evol. 41:37.

Vallimont, J. E., C. D. Dechow, J. M. Daubert, M. W. Dekleva, J. W. Blum, W. Liu, G. A. Varga, A. J. Heinrichs, and C. R. Baumrucker. 2013. Short communication: Feed utilization and its associations with fertility and productive life in 11 commercial Pennsylvania tie-stall herds. J. Dairy Sci. 96:1251-1254.

VanRaden, P. M., C. P. Van Tassell, G. R. Wiggans, T. S. Sonstegard, R. D. Schnabel, J. F. Taylor, and F. S. Schenkel. 2009. Invited review: Reliability of genomic predictions for North American Holstein bulls. J. Dairy Sci. 92:16-24.

Varga, G., and C. Dechow. 2013. Can we use residual feed intake to enhance dairy production efficiency? Tri-State Dairy Nutrition Conference. Accessed Mar. 14, 2016. https://www.cabdirect.org/ cabdirect/abstract/20133232173.

Veerkamp, R. F., J. E. Pryce, D. Spurlock, D. Berry, M. Coffey, P. Løvendahl, R. van der Linde, J. Bryant, F. Miglior, Z. Wang, M. Winters, N. Krattenmacher, N. Charfeddine, J. Pedersen, and Y. de Haas. 2013. Selection on feed intake or feed efficiency: A position paper from gDMI breeding goal discussions. Interbull Bull. $47: 15-22$.

Williams, Y. J., J. E. Pryce, C. Grainger, W. J. Wales, N. Linden, M Porker, and B. J. Hayes. 2011. Variation in residual feed intake in Holstein-Friesian dairy heifers in southern Australia. J. Dairy Sci. 94:4715-4725.

Wolc, A., J. Arango, P. Settar, J. E. Fulton, N. P. O'Sullivan, R. Preisinger, D. Habier, R. Fernando, D. J. Garrick, and J. C. M. Dekkers. 2011. Persistence of accuracy of genomic estimated breeding values over generations in layer chickens. Genet. Sel. Evol. 43:23.

Wright, S. 1931. Evolution in Mendelian populations. Genetics 16:97159.

Yao, C., G. de los Campos, M. J. VandeHaar, D. M. Spurlock, L. E. Armentano, M. Coffey, Y. de Haas, R. F. Veerkamp, C. R. Staples, E. E. Connor, Z. Wang, M. D. Hanigan, R. J. Tempelman, and K. A. Weigel. 2017. Use of genotype $\mathrm{x}$ environment interaction model to accommodate genetic heterogeneity for residual feed intake, dry matter intake, net energy in milk, and metabolic body weight in dairy cattle. J. Dairy Sci. 100:2007-2016. 